\title{
Psicooncología
}

ISSN: 1696-7240

\section{La influencia del estrés y el optimismo sobre la calidad de sueño de mujeres con cáncer de mama}

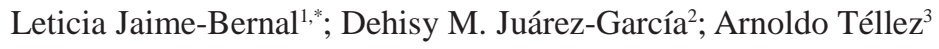

Recibido: 17 de noviembre de 2017 / Aceptado: 20 de agosto de 2018

Resumen: Objetivo: El objetivo de este estudio fue determinar la influencia del estrés y el optimismo sobre la calidad de sueño de mujeres con cáncer de mama. Método: Diseño trasversal correlacional. Los instrumentos utilizados fueron el Índice de Calidad del Sueño de Pittsburgh, la Escala de Estrés Percibido y la Escala de Orientación de Vida. Participaron 60 pacientes con cáncer de mama en estadios tempranos, sin metástasis, con una media de edad de 51,4 $( \pm 9,3)$ años. Resultados: Las pacientes obtuvieron una media de 7,5 $( \pm 4,4)$ del Índice de calidad de sueño global, en donde a mayor puntaje indica una mala calidad de sueño, una media de $24,5( \pm 9,5)$ para estrés y una media 37,6 $( \pm 5,5)$ para optimismo. Se obtuvo una correlación positiva y significativa entre el estrés y calidad de sueño global $(\mathrm{r}=0,349, \mathrm{p}=0.006)$, y una correlación negativa y significativa entre optimismo y calidad de sueño $\left(r_{s}^{s}=-0,524, p=0,001\right)$. El optimismo predice la calidad de sueño global en un 25,8\%. Conclusión: En mujeres con cáncer de mama en estadios tempranos, la relación entre variables indica que a mayor estrés menor calidad de sueño, menor duración del dormir y más alteraciones de sueño. En cuanto a la variable de optimismo, indica que, a mayor optimismo, mayor calidad subjetiva de sueño, mayor duración, menor latencia de sueño y menos alteraciones del dormir. Por lo tanto, se concluye que, en mujeres con cáncer de mama, el estrés reduce la calidad de sueño mientras que el optimismo la beneficia directamente.

Palabras clave: Calidad de sueño; optimismo; estrés; cáncer de mama.

\section{[en] The influence of stress and optimism on sleep quality in women with breast cancer}

Abstract: Objective: The purpose of this study was to determine the influence of stress and optimism on the quality of sleep of women with breast cancer. Method: Cross-sectional correlational design. The instruments used were the Pittsburgh Sleep Quality Index, Perceived Stress Scale, and Life Guidance Scale. Participated in 60 patients with early stage of breast cancer, with no metastasis and an average

1 Leticia Jaime-Bernal. Centro de Investigación y Desarrollo en Ciencias de la Salud, CIDICS. Facultad de Psicología de la Universidad Autónoma de Nuevo León, UANL. México.

E-mail: ly_jaime@hotmail.com

2 Dehisy M. Juárez-García. Centro de Investigación y Desarrollo en Ciencias de la Salud, CIDICS. Facultad de Psicología de la Universidad Autónoma de Nuevo León, UANL. México

E-mail: dehisy_jg@yahoo.com.mx

3 Arnoldo Téllez Centro de Investigación y Desarrollo en Ciencias de la Salud, CIDICS. Facultad de Psicología de la Universidad Autónoma de Nuevo León, UANL. México

E-mail: arnoldo.tellez@uanl.mx

* Correspondencia: Leticia Jaime-Bernal. Facultad de Psicología de la Universidad Autónoma de Nuevo León, UANL. Campus Ciencias de la Salud. Centro de Investigación y Desarrollo en Ciencias de la Salud (CIDICS). Av. Carlos Canseco s/n con Av. Gonzalitos, Mitras Centro Monterrey, Nuevo León, México, C.P. 64460.

E-mail: ly_jaime@hotmail.com,psalud.cidics@gmail.com 
age of $51.4( \pm 9.3)$ years. Results: The patients obtained a mean of $7.5( \pm 4.4)$ overall sleep quality index, where a higher score indicates a poor sleep quality, with a mean of $24.5( \pm 9.5)$ for stress and a mean $37.6( \pm 5.5)$ for optimism. There was a positive and significant correlation between stress and overall sleep quality $(\mathrm{rs}=0.349, \mathrm{p}=0.006)$, and a negative and significant correlation between optimism and sleep quality $(\mathrm{rs}=-0.524, \mathrm{p}=0.001)$. The optimism predicts the overall sleep quality by $25.8 \%$. Conclusion: In women with breast cancer in the early stages, the relationship between variables indicates that higher stress lower quality of sleep, shorter sleep duration and more sleep disturbances. Regarding the variable of optimism, indicates that to greater optimism, higher subjective quality of sleep, longer duration, and lower sleep latency and sleep disturbances. Therefore, it is concluded that in women with breast cancer, stress reduces sleep quality while optimism benefits directly.

Keywords: Sleep quality; optimism; stress; breast cancer.

Sumario. 1. Introducción 2. Método 2.1. Participantes 2.2. Instrumentos 2.2.1. Índice de Calidad del Sueño de Pittsburgh 2.2.2. Escala de Estrés Percibido 2.2.3. Escala de Orientación de vida 2.3. Procedimiento 3. Análisis de datos 4. Resultados 5. Discusión 6. Conclusión 7. Referencias bibliográficas.

Cómo citar: Jaime-Bernal L, Juárez-García DM, Téllez A. La influencia del estrés y el optimismo sobre la calidad de sueño de mujeres con cáncer de mama. Psicooncología 2018;15:249-260. doi: 10.5209/PSIC.61434.

\section{Introducción}

El sueño es indispensable para el buen funcionamiento cognitivo y emocional en los seres humanos, y sus alteraciones o su privación se asocian a la reducción de la calidad de $\operatorname{vida}^{(1)}$. Existe una correlación entre los procesos de sueño y el estado general de salud(física y emocional), y las enfermedades crónicas, ya que éstas suelen modificar los patrones de sueño o en ocasiones, precisamente son los cambios anormales en la cantidad y calidad de sueño, los que pueden generar estas enfermedades ${ }^{(2)}$.

Para las mujeres con cáncer de mama, las dificultades para dormir suelen representar un gran problema, puesto que la quimioterapia y/o radioterapia frecuentemente afectan su calidad de sueño(3). Estas alteraciones de sueño, a su vez se vinculan con un deterioro en el funcionamiento físico y cognitivo, y con una menor calidad de vida ${ }^{(4)}$.

El aumento en el malestar psicológico como el estrés, la depresión, la desesperanza y la calidad de vida son factores predictivos para desarrollar problemas de sueño en pacientes con cáncer ${ }^{(5,6)}$, ya que se ha encontrado que tener una respuesta adversa o desadaptada ante el diagnóstico de la enfermedad, se asocia con la interrupción de los ritmos circadianos del sueño-vigilia ${ }^{(7)}$. El estrés y el estado de ánimo son factores que pueden contribuir a estos ritmos biológicos anormales, y por lo tanto pueden afectar la calidad de sueño ${ }^{(8)}$.

En el ser humano existe el estado de vigilia, y cuatro fases de sueño propuestas por la Academia Americana de Medicina del Sueño, que son las siguientes: Etapa W (vigilia por sus siglas en inglés), Fase N1, Fase N2, Fase N3 que corresponde al sueño No REM y la Fase R, que corresponde al sueño REM ${ }^{(9)}$.

Las condiciones de estrés durante la vigilia, modifican la cantidad y la calidad de sueño ${ }^{(10)}$. A nivel biológico, un estresor puede provocar una descarga de adrenalina y cortisol que mantienen al cerebro en un estado activado de vigilia, 
dificultando conciliar el sueño(11) , alterando su duración; y la fase asociada a Movimientos Oculares Rápidos (REM), la cual se encarga de integrar las memorias angustiantes, por lo que el sueño funge un papel importante en la reducción del estrés y viceversa ${ }^{(6)}$.Existe una fuerte correlación entre el estrés y la aparición del insomnio y otros trastornos del sueño ${ }^{(12)}$; Mauss, Troy y LeBourgeois ${ }^{(13)}$ han encontrado que una pobre capacidad para regular las emociones negativas en el estado de vigilia se asocia a una mala calidad de sueño.

La importancia del sueño nocturno para la salud física y mental ha sido ampliamente reconocida y estudiada en los últimos tiempos, como también, el estudio de las características positivas de la personalidad como el optimismo y la autoestima, debido a su relación con el bienestar subjetivo y la salud ${ }^{(14)}$. Algunas investigaciones han encontrado que las personas que tienen alto nivel del bienestar, felicidad y emociones positivas, tienen mejor salud ${ }^{(15)}$.

El optimismo es un rasgo básico de la personalidad positiva y se presenta en las pacientes que no perciben tan afectada su calidad de vida y tiene relación con variables como calidad de sueño, depresión, energía, estrés y optimismo disposicional ${ }^{(16)}$. El optimismo disposicional es una variable de diferencia individual que manifiesta la medida en que las personas tienen expectativas generalizadas favorables para su futuro ${ }^{(17)}$.

Henselmans et al. ${ }^{(18)}$ encontraron que una de cada tres mujeres con cáncer de mama tenía una actitud positiva y esto favorecía la tolerancia al tratamiento. Por ejemplo, las mujeres que pensaban que se podrían curar del cáncer manifestaron características autoprotectoras. Asimismo Mera y Ortiz ${ }^{(19)}$, coinciden con que el optimismo mejora la calidad de vida, generando que las pacientes se adapten más fácilmente a la enfermedad, disminuyendo el miedo y ansiedad asociados al diagnóstico y tratamiento del cáncer, y protegiéndolas ante complicaciones físicas y emocionales asociadas a la enfermedad.

Las mujeres con niveles de optimismo más altos, experimentaban menos estrés antes y después del diagnóstico de cáncer de mama que aquellas con niveles de optimismo más bajos. El optimismo tiene un efecto amortiguador durante el estrés psicológico experimentado por las mujeres antes y después del diagnóstico y puede ser una herramienta útil en la reducción de eventos estresantes de la vida. Dentro de la condición estresante de la enfermedad, el optimismo mejora las habilidades personales para hacer frente a la situación de estrés ${ }^{(20,21)}$.

Schou, Ekeberg, Sandvik, y Ruland ${ }^{(22)}$ en un estudio longitudinal examinaron la relación entre el optimismo, la calidad de vida y estrategias de supervivencia de 165 mujeres diagnosticadas con cáncer de mama y sus resultados mostraron que las mujeres optimistas respondieron al diagnóstico y al tratamiento, con espíritu de lucha, que a su vez tuvo un efecto positivo en su calidad de vida, mientras que las mujeres pesimistas respondieron con desesperanza, lo que tuvo un impacto negativo en su calidad de vida. Sohl et al. ${ }^{(23)}$ encontraron que las mujeres que eran menos pesimistas se sentían mejor emocionalmente al principio del tratamiento y las mujeres que estaban más estresadas durante el tratamiento se sentían peor emocionalmente al principio. También se ha encontrado que la reducción del estrés tiene un potencial clínicamente valioso para mejorar el estado de ánimo y la calidad de sueño en pacientes de cáncer ${ }^{(24)}$. 
Debido a lo anterior, se espera encontrar que niveles más altos de optimismo y menores niveles de estrés favorecerán la calidad de sueño, mientras que, por el contrario, cuando se presenta menor nivel de optimismo y un mayor nivel de estrés aumentarán los problemas de sueño. El objetivo de este estudio es determinar la influencia del estrés y el optimismo sobre la calidad de sueño en mujeres con cáncer de mama.

\section{Método}

El diseño de este estudio fue transversal-correlacional( ${ }^{(25)}$.

\subsection{Participantes}

Los criterios de inclusión de las participantes fueron: pacientes con diagnóstico de cáncer de mama en estadios tempranos, previo al inicio del tratamiento médico para el cáncer. Los criterios de exclusión fueron cáncer bilateral o cáncer previo, algún otro tipo de cáncer o estadios avanzados con metástasis. Participaron 60 pacientes con cáncer de mama con una media de edad de 51,4( $\pm 9,3)$ años. La mayoría era casada o vivía en pareja $(61,7 \%)$, el $85 \%$ tenía hijos y el estatus socioeconómico era de nivel medio-bajo.

\subsection{Instrumentos}

\subsection{1. Índice de Calidad del Sueño de Pittsburgh}

(Pittsburgh Sleep Quality Index, PSQI) elaborado por Buysse, Reynolds, Monk, Berman y Kupfer ${ }^{(26)}$, el cual proporciona una puntuación global de la calidad de sueño y puntuaciones parciales en siete componentes distintos: calidad subjetiva del sueño, latencia, duración, eficiencia habitual, alteraciones, uso de medicación hipnótica y disfunción diurna. Cuenta con un coeficiente de alfa de Cronbach de 0,67 y 0,81 . En este estudio se utilizó la versión en español de Jiménez-Genchi, Monteverde-Maldonado, Nenclares-Portocarrero, Esquivel-Adame y de la VegaPacheco(27) que cuenta con un alfa de Cronbach de 0,78. El rango del puntaje global es de 0 a 21, una puntuación global mayor a 5 indica una mala calidad de sueño.

\subsubsection{Escala de Estrés Percibido}

(Perceived Stress Scale, PSS) fue elaborada por Cohen, Kamarak y Mermelstein ${ }^{(28)}$ para evaluar el grado en que las situaciones de la vida son valoradas como estresantes, obteniendo un alfa de Cronbach de 0,75. En este estudio se utilizó la adaptación cultural realizada en México por González Ramírez y Landero Hernández ${ }^{(29)}$, la cual cuenta con 14 ítems y un coeficiente de alfa de Cronbach de 0,83 , además de confirmar la estructura factorial de la escala original, utilizando un análisis factorial confirmatorio. A mayor puntuación, mayor estrés percibido. 


\subsubsection{Escala de Orientación de vida}

(Life Orientation Test, LOT-R) de Scheier, Carver y Bridges ${ }^{(30)}$ consta de 10 ítems en escala Lickert de 5 puntos. Seis de los ítems miden el optimismo disposicional y los otros 4 sirven para hacer menos evidente el contenido del test; esta escala cuenta con una fiabilidad adecuada $(\alpha=0,79)$. Se utilizó la versión española de Otero, Luengo, Romero, Gómez y Castro ${ }^{(31)}$ del LOT-R. De los 6 ítems de optimismo disposicional, 3 están redactados en sentido positivo (optimismo) y 3 en sentido negativo (pesimismo). Las puntuaciones de los ítems redactados en sentido negativo se invierten y se obtiene una puntuación total orientada hacia el polo de optimismo.

\subsection{Procedimiento}

Este estudio forma parte de una investigación más amplia en mujeres con cáncer de mama que fue aprobada por el Comité de Bioética del Centro de Investigación y Desarrollo en Ciencias de la Salud.

Las pacientes fueron contactadas a través de la Clínica de Mama del Hospital Metropolitano "Bernardo Sepúlveda" y referidas por un Oncólogo del Instituto Mexicano del Seguro Social.

Se tomaron los datos de las pacientes que cumplían con los criterios de inclusión, fueron contactadas para invitarlas al estudio, las que aceptaron la invitación firmaron un consentimiento informado y posteriormente respondieron los cuestionarios de evaluación.

\section{Análisis de datos}

Los datos fueron analizados con el paquete estadístico SPSS versión 21. Se realizaron los análisis descriptivos de las variables y la prueba de normalidad, de la cual se obtuvieron resultados significativos para la mayoría de las variables, por lo que se decidió utilizar pruebas no paramétricas. Para el análisis de correlación de utilizó el coeficiente de Spearman.

Debido al número de pares de correlaciones se utilizó la corrección de Bonferroni para evitar el incremento de la probabilidad de error tipo $\mathrm{I}^{(32)}$, por lo que para este estudio se consideraron estadísticamente significativas solo las variables que obtuvieron un valor de $p$ igual o menor a 0,007 . Para el análisis de regresión múltiple se utilizó el método de pasos sucesivos para evaluar las variables que más influyen en la calidad de sueño.

Tamaño del efecto

La American Psychological Association ${ }^{(33)}$ recomienda ampliamente el uso del tamaño del efecto junto con la $p$ de las pruebas estadísticas inferenciales al momento de reportar los resultados. Habitualmente se utiliza el coeficiente de correlación ( $r$ de Pearson) para medir el grado de la fuerza y dirección (negativa o positiva) de asociación entre dos variables, lo que se considera en sí mismo un tamaño del efecto. Para interpretar el tamaño del efecto mediante $r$ se debe 
considerar que a mayor correlación, mayor es el tamaño del efecto que la variable $X$ generó sobre la variable dependiente $Y$. Cohen ${ }^{(34)}$, estableció para una correlación de $r=0,10$ o más como un tamaño del efecto pequeño, para una $r=0,30$ o más un tamaño del efecto mediano, y para una $r$ igual o mayor a 0,50 un tamaño del efecto grande, lo que actualmente se considera como un convencionalismo entre los investigadores ${ }^{(35)}$.

\section{Resultados}

En los análisis descriptivos las pacientes obtuvieron una media de 7,5 $( \pm 4,4)$ del índice de calidad de sueño global, una media de $24,5( \pm 9,5)$ para estrés y una media $37,6( \pm 5,5)$ para optimismo (Tabla 1$)$.

Tabla 1. Análisis descriptivo de las variables

\begin{tabular}{lcc}
\hline Variable & Rango de puntaje de la escala & Media (DT) \\
\hline Calidad de sueño* & $0-21$ & $7,5(4,4)$ \\
Calidad subjetiva & $0-3$ & $1,1(, 72)$ \\
Latencia & $0-3$ & $1,1(1,1)$ \\
Duración & $0-3$ &, $76(, 98)$ \\
Eficiencia & $0-3$ & $1,0(1,2)$ \\
Alteraciones & $0-3$ & $1,4(, 56)$ \\
Uso medicamento & $0-3$ &, $45(, 44)$ \\
Disfunción diurna & $0-3$ & $1,5(, 99)$ \\
Estrés & $0-56$ & $24(9,5)$ \\
Optimismo & $10-50$ & $37,6(5,5)$ \\
\hline
\end{tabular}

*Calidad de sueño y sus componentes: a mayor puntaje, menor calidad de sueño.

En el análisis muestra una correlación negativa significativa entre el estrés y calidad de sueño global, esto indica que, a mayor estrés, peor calidad de sueño. También se observa que, a mayor nivel de optimismo, mejor calidad de sueño (ver tabla 2).

En cuanto a la relación entre el estrés y el optimismo con los componentes de la calidad de sueño del PSQI, se observa que a mayor estrés menor duración de sueño y más alteraciones de sueño. Referente al optimismo se encontró que a mayor optimismo mejor calidad subjetiva de sueño, mayor duración de sueño, menor latencia de sueño y menos alteraciones de sueño (Tabla 2).

Como se puede observar, existe un tamaño del efecto grande entre las variables de optimismo y calidad subjetiva de sueño $(r=-0,55)$, alteraciones de sueño $(r=-0,55)$ y calidad global de sueño $(\mathrm{r}=-0,52)$. 
En la regresión múltiple con el índice de calidad de sueño global como variable dependiente, y el estrés y el optimismo como variables independientes, se obtuvo un modelo en el cual la variable de optimismo predice la calidad de sueño global en un $25,7 \%$ con un cambio de $\mathrm{F}$ significativo $(p<0,01)$. El coeficiente de regresión fue 0,520 , el coeficiente Beta del optimismo fue negativo y significativo lo que indica que a mayor optimismo mejor calidad de sueño.

Tabla 2. Análisis de correlación entre las variables optimismo, estrés y componentes del PSQI

\begin{tabular}{lllllllll}
\hline $\begin{array}{c}\text { Componentes } \\
\text { PSQI }\end{array}$ & $\begin{array}{c}\text { Calidad } \\
\text { Subjetiva }\end{array}$ & Latencia & Duración & Eficacia & Alteraciones & $\begin{array}{c}\text { Uso de } \\
\text { Medica- } \\
\text { mento }\end{array}$ & $\begin{array}{c}\text { Disfunción } \\
\text { Diurna }\end{array}$ & $\begin{array}{c}\text { Calidad } \\
\text { Sueño } \\
\text { Global }\end{array}$ \\
\hline Estrés &, 331 &, 211 &, $357^{*}$ &, 259 &, $354^{*}$ &,- 019 &, 318 &, $349^{*}$ \\
Optimismo & $\mathbf{- , 5 5 2 *}$ &,$- 458^{*}$ &,$- 344^{*}$ &,$- 262^{*}$ & $\mathbf{- , 5 5 1 *}$ &,- 083 &,- 269 & $\mathbf{- , 5 2 4 *}$ \\
\hline
\end{tabular}

$* p<, 007$. Las variables resaltadas en negrita representan un tamaño del efecto grande

Tabla 3. Regresión Múltiple

\begin{tabular}{lccccc}
\hline Calidad de sueño global & $\mathrm{R}^{2}$ & $\mathrm{R}^{2 \text { aj }}$ & $\mathrm{B}$ & Beta & $t$ \\
\hline $\begin{array}{l}\text { Modelo } 1 \\
\text { Optimismo }\end{array}$ &, 271 &, 258 &,- 410 &,- 520 & $-4,63^{* *}$ \\
$* * * p, 01$ & & & & & \\
\hline
\end{tabular}

\section{Discusión}

La calidad de sueño es muy importante para el buen funcionamiento cognitivo y social en la vida diaria. Durante el sueño se secretan hormonas importantes, como la hormona del crecimiento y la melatonina, que favorecen la restauración celular y tienen efectos antioxidantes y antineoplásicos ${ }^{(36)}$. Además durante el sueño se favorece el traslado de la información adquirida durante el día de la memoria de corto a largo plazo ${ }^{(37)}$. Cuando existe privación de sueño, se produce una disminución en el conteo de las células del sistema inmune y se puede desencadenar un estado depresivo y pensamientos negativos que afectan aún más la interrupción del sueño, ya que el insomnio está altamente correlacionado con estrés y sentimientos negativos ${ }^{(38,39)}$. En los análisis descriptivos las pacientes obtuvieron una media de 7,5 del índice de calidad de sueño global en el PSQI, lo cual coincide con lo reportado en otras muestras de pacientes con cáncer de mama $^{(40)}$ este puntaje indica una mala calidad de sueño según el punto de corte de 5 establecido en población general( ${ }^{(27)}$. 
Respecto al estrés, el promedio del puntaje obtenido fue de 24 , y es similar al encontrado en mujeres de población general mexicana ${ }^{(41)}$. Por otra parte el puntaje promedio de optimismo fue de 35 , el cual es alto considerando el puntaje de 22 obtenido en una muestra española de pacientes con cáncer ${ }^{(42)}$.

En cuanto a la hipótesis principal, se esperaba encontrar una relación entre el optimismo y el estrés que tuvieran un impacto en la calidad de sueño, es decir, que al elevarse el nivel de optimismo, el nivel de estrés disminuiría y por ende mejoraría la calidad de sueño. Sin embargo, en este estudio se observa una relación directa del optimismo disposicional que impacta positivamente en la calidad de sueño sin mediación del estrés, esto quiere decir que, a mayor nivel de optimismo, mejor calidad global de sueño en pacientes con cáncer de mama, independientemente del nivel de estrés que presenten.

Esto coincide con los resultados del estudio de Norlander, Johansson y Bood ${ }^{(16)}$ que indican que las personas optimistas tienen la capacidad de usar el estrés para beneficio, como afrontamiento y tener mayor probabilidad de éxito, las pacientes que muestran mayor nivel de optimismo reportan mayores niveles de energía y mejor calidad de sueño, esto incluso teniendo altos niveles de estrés, concluyendo que el optimismo disposicional parece ser un rasgo básico y estable de la persona que no se ve afectada fácilmente por su salud.

Lemola, Räikkönen, Gómez y Allemand ${ }^{(14)}$ mostraron que el optimismo se asoció negativamente con los síntomas del insomnio. Existe evidencia de que características de personalidad positiva, tales como el optimismo y la autoestima son importantes predictores de buena salud y favorecen la calidad y la duración de sueño.

En el presente estudio también se encontró que el optimismo se correlaciona con una mejor calidad de sueño subjetiva, mayor duración de sueño, menor latencia de sueño y alteraciones de sueño, dando lugar a una mejor calidad de sueño global. La calidad de sueño subjetiva es la que más se correlaciona con optimismo, siendo esta uno de los componentes importantes, ya que indica una mejoría en la percepción de buen sueño y, por lo tanto, de buena salud. Es importante mencionar que no se encontró relación entre el optimismo y el estrés, como era lo esperado, sin embargo, coincide con otros estudios que indican que las personas que presentan niveles altos de estrés y ansiedad, pero también presentan niveles altos de optimismo, pueden desarrollar estrategias de afrontamiento positivas y dominar el estrés consiguiendo una buena calidad de vida y calidad de sueño ${ }^{(16)}$.

Las personas que tienen expectativas positivas sobre el futuro, presentan menos problemas de sueño, sin embargo, las que piensan negativamente presentan una calidad de sueño insuficiente, ya que parece ser, que los pensamientos interfieren durante la noche ${ }^{(38)}$. También se ha encontrado que en altos niveles de optimismo, se correlaciona además de una buena calidad de sueño, con una mejor adaptación a sus actividades del hogar y menos efectos negativos, como el miedo, la ira, el dolor, dificultades de movimiento y cansancio ${ }^{(42)}$.

Sin embargo, en cuanto a la relación con la variable de estrés, se observa una correlación positiva y significativa entre estrés y calidad de sueño global, esto indica que a mayor estrés peor calidad de sueño, esta correlación también se ha comprobado en estudios como el de García, Querts, Hierresuelo y Quesada ${ }^{(12)}$, en el que se menciona que el estrés tiene una relación directa con la aparición de insomnio y problemas para dormir. También en los resultados de Akechi et 
al. ${ }^{(5)}$ que indican que el aumento de malestar psicológico es el factor predictivo en desarrollo de alteraciones de sueño en las pacientes con cáncer. Los problemas de sueño se asocian a varias comorbilidades incluyendo complicaciones, hipertensión, depresión, ansiedad o estrés que afectan el desarrollo de la enfermedad y empeoran la calidad de vida ${ }^{(43)}$.

Los pensamientos intrusivos o negativos y los factores psicológicos están relacionados con los problemas del sueño, se asocian con la duración y la eficiencia del sueño ${ }^{(11)}$, que coincide con los resultados de este estudio en el que se menciona que el optimismo se asocia con mayor duración y menos alteraciones de sueño, por lo que se recomienda para futuros estudios realizar intervenciones para incrementar el optimismo, para obtener como resultado la mejora de la calidad de sueño.

Considerando los resultados, se podría recomendar la realización de intervenciones dirigidas al incremento del optimismo en pacientes con diagnóstico de cáncer de mama. Algunas de las intervenciones que han demostrado ser efectivas para incrementar el optimismo y la calidad de sueño, han sido la hipnoterapia ${ }^{(44,45)}$ y técnicas cognitivas, tales como la creación de sentido ${ }^{(46)}$. Sin embargo, también se considera que es necesario realizar mayor investigación, con diferentes tipos y tamaños de muestra para comprobar que los resultados se mantengan.

\section{Conclusión}

El optimismo es una característica positiva que influye en forma importante en la calidad de sueño de las pacientes diagnosticadas con cáncer de mama, aumentando la duración del sueño, acortando la latencia (tiempo que tarda para conciliar el sueño) y disminuyendo las alteraciones de sueño, sin estar relacionado al nivel de estrés. Aunque, por otra parte, el estrés empeora la calidad de sueño, acortando su duración y presentando más alteraciones de sueño.

Se recomienda diseñar y evaluar nuevas intervenciones psicosociales que favorezcan el aumento del optimismo en las pacientes con cáncer de mama, para beneficiar su calidad de sueño y, por lo tanto, su calidad de vida, también se recomienda como también replicar este estudio en otras poblaciones para comprobar los resultados.

\section{Referencias bibliográficas}

1. Franco-Pérez J, Ballesteros-Zebadúa P, Custodio V, Paz C. Principales neurotransmisores involucrados en la regulación del ciclo sueño-vigilia. Revista de Investigación Clínica 2012; 64: 182-91.

2. Miró E, Cano-Lozano MC, Buela-Casal G. Sueño y calidad de vida. Revista Colombiana de Psicología 2005; 14: 11-27.

3. Ewertz M, Jensen AB. Late effects of breast cancer treatment and potentials for rehabilitation. Acta Oncol 2011; 50:187-93. doi:10.3109/0284186X.2010.533190

4. Berger AM, Wielgus K, Hertzog M, Fischer P, Farr L. Patterns of circadian activity rhythms and their relationships with fatigue and anxiety/depression in women treated with breast cancer adjuvant chemotherapy. Support Care Cancer 2010; 18: 105-14. doi: $10.1007 / \mathrm{s} 00520-009-0636-0$ 
5. Akechi T, Okuyama T, Akizuki N, Shimizu K, Inagaki M, Fujimori M, et al. Associated and predictive factors of sleep disturbance in advanced cancer patients. Psychooncology 2007; 16: 888-94. doi: 10.1002/pon.1122

6. Mystakidou K, Parpa E, Tsilika E, Gennatas C, Galanos A, Vlahos L. How is sleep quality affected by the psychological and symptom distress of advanced cancer patients? Palliat Med 2009; 23: 46-53. doi: 10.1177/0269216308098088.

7. Dedert E, Lush E, Chagpar A, Dhabhar FS, Segerstrom SC, Spiegel D, et al. Stress, coping, and circadian disruption among women awaiting breast cancer surgery. Ann Behav Med 2012; 44: 10-20. doi: 10.1007/s12160-012-9352-y.

8. Spiegel D, Sephton, SE. Psychoneuroimmune and endocrine pathways in cancer: effects of stress and support. Semin Clin Neuropsychiatry 2001; 6: 252-65. doi: 10.1053/ scnp.2001.26995

9. Silber M H, Ancoli-Israel S, Bonnet MH, Chokroverty S, Grigg-Damberger MM, Hirshkowitz et al. The visual scoring of sleep in adults. J Clin Sleep Med 2007; 3: 121-31.

10. Cano-Lozano MC, Espinosa-Fernández L, Miró E, Buela-Casal G. Una revisión de las alteraciones del sueño en la depresión. Rev Neurol 2003; 36: 366-75.

11. Wright CE, Schnur JB, Montgomery GH, Bovbjerg, DH. Psychological factors associated with poor sleep prior to breast surgery: an exploratory study. Behav Med 2010; 36: 85-91. doi: 10.1080/08964280903521305

12. García Gascón A, Querts Méndez O, Hierresuelo Izquierdo EJ, Quesada Vidal S. Algunos aspectos psicosociales del insomnio en estudiantes de primer año de medicina. Medisan 2015; 19:56-60

13. Mauss IB, Troy AS, LeBourgeois MK. Poorer sleep quality is associated with lower emotion-regulation ability in a laboratory paradigm. Cognition Emotion 2013; 27: 567 76. doi:10.1080/02699931.2012.727783

14. Lemola S, Räikkönen K, Gómez V, Allemand M. Optimism and Self-Esteem Are Related to Sleep. Results from a large community-based sample. Inter J Behav Med 2013; 20: 567-71. doi: 10.1007/s12529-012-9272-z

15. Vera-Villarroel P, Celis-Atenas K. Afecto positivo y negativo como mediador de la relación optimismo-salud: evaluación de un modelo estructural. Universitas Psychologica 2014; 13: 1017-26. doi: 10.11144/Javeriana.UPSY13-3.apnm

16. Norlander T, Johansson A, Bood SA. The affective personality: its relation to quality of sleep, well-being and stress. Soc Behav Pers 2005; 33: 709-22.

17. Carver CS, Scheier MF, Segerstrom SC. Optimism. Clin Psychol Rev 2010; 30: 879-89. doi:10.1016/j.cpr.2010.01.006

18. Henselmans I, Helgeson VS, Seltman H, de Vries J, Sanderman R, Ranchor AV. Identification and prediction of distress trajectories in the first year after a breast cancer diagnosis. Health Psychol 2010; 29: 160-8. doi: 10.1037/a0017806

19. Mera PC, Ortiz M. La relación del optimismo y las estrategias de afrontamiento con la calidad de vida de mujeres con cáncer de mama. Terapia Psicológica 2012; 30: 69-78.

20. Shaheen N, Andleeb S, Ahmad S, Bano A. Effect of optimism on psychological stress in breast cancer women. J Soc Sci 2014; 8: 84-90.

21. Karademas EC, Karvelis S, Argyropoulou K. Stress-related predictors of optimism in breast cancer survivors. Stress Health 2007; 23: 161-8. doi: 10.1002/smi.1132.

22. Schou I, Ekeberg Ø, Sandvik L, Ruland CR. Stability in optimism-pessimism in relation to bad news: A study of women with breast cancer. J Pers Assess 2005; 84: 148-54. doi: 10.1207/s15327752jpa8402_04 
23. Sohl SJ, Schnur JB, Sucala M, David D, Winkel G, Montgomery GH. Distress and emotional well-being in breast cancer patients prior to radiotherapy: an expectancybased model. Psychol Health 2012; 27: 347-61. doi: 10.1080/08870446.2011.569714

24. Smith JE, Richardson J, Hoffman C, Pilkington K. Mindfulness-Based Stress reduction as supportive therapy in cancer care: systematic review. J Adv Nurs 2005; 52: 315-27. doi: 10.1111/j.1365-2648.2005.03592.x

25. Urdiales Ibarra ME, Leyva Torres P, Villarreal Peña G. Diseños de investigación no experimentales. En: Landero-Hernández R, González-Ramírez MT, editores. Estadística con SPSS y metodología de la investigación. México D.F: Trillas, 2007.

26. Buysse DJ, Reynolds III CF, Monk TH, Berman SR, Kupfer DJ. The Pittsburgh sleep quality index: A new instrument for psychiatric practice and research. Psychiatry Res 1989; 28:193-213. doi: 10.1016/0165-1781(89)90047-4.

27. Jiménez-Genchi A, Monteverde-Maldonado E, Nenclares-Portocarrero A, EsquivelAdame G, de la Vega-Pacheco A. Confiabilidad y análisis factorial de la versión en español del Indice de Calidad de Sueño de Pittsburgh en pacientes psiquiátricos. Gaceta Médica de México 2008; 144: 491-496.

28. Cohen S, Kamarck T, Mermelstein R. A global measure of perceived stress. J Health Soc Behav 1983; 24: 385-96.

29. González Ramírez MT, Landero Hernández R. Factor structure of the perceived Stress Scale (PPS) in a Sample from Mexico. Spanish J Psychol 2007; 10: 199-206.

30. Scheier MF, Carver CS, Bridges MW. Distinguishing optimism from neuroticism (and trait anxiety, self-mastery, and self-esteem): a reevaluation of the Life Orientation Test. J Pers Soc Psychol 1994; 67: 1063-78. doi 10.1037/0022-3514.67.6.1063

31. Otero JM, Luengo A, Romero E, Gómez Fraguela JA, Castro C. Psicología de la personalidad; Manual de prácticas. Barcelona: Ed. Ariel Practicum, 1998.

32. Armstrong RA. When to use the Bonferroni correction. Ophthalmic Physiol Opt 2014; 34: 502-8. doi: 10.1111/opo.12131.

33. American Psychological Association. Publication manual of American psychological association. Washington D.C: Author, 2010.

34. Cohen J. The power primer. Psychol Bull 1992; 112: 155-9. doi: 10.1037/00332909.112.1.155

35. Hemphill JF. Interpreting the magnitudes of correlation coefficients. Am Psychol 2003; 58: 78-9. doi: 10.1037/0013164402062002002.

36. Blask DE. Melatonin, sleep disturbance and cancer risk. Sleep Med Rev 2009; 13: $257-$ 64. doi: 10.1016/j.smrv.2008.07.007

37. Téllez A. Atención, aprendizaje y memoria: aspectos psicobiológicos. México: Trillas, 2002.

38. Sing CY, Wong WS. Prevalence of insomnia and its psychosocial correlates among college students in Hong Kong. J Am Coll Health 2010; 59: 174-82. doi: 10.1080/07448481.2010.497829.

39. Irwin MR. Why sleep is important for health: A psychoneuroimmunology perspective. Ann Rev Psychol 2015; 66: 143-72. doi: 10.1146/annurev-psych-010213-115205

40. Carpenter JS, Andrykowski MA. Psychometric evaluation of the Pittsburgh sleep quality index. J Psychosom Res 1998; 45: 5-13.

41. González-Ramírez MT, Rodríguez-Ayán MN, Landero Hernández R. The Perceived Stress Scale (PSS): Normative data and factor structure for a large-scale sample in Mexico. Spanish J Psychol 2013; 16: E47. doi: 10.1017/sjp.2013.35. 
42. Pastells Pujol S, Font Guiteras A. Optimismo disposicional y calidad de vida en mujeres con cáncer de mama. Psicooncología 2014; 11: 19-29. doi: 10.5209/rev_PSIC.2014. v11.n1.44914

43. Brann L, Owens J, Williamson, A. The handbook of contemporary clinical hypnosis: theory and practice. Oxford UK: Wiley-Blackwell, 2012.

44. Téllez López A, Rodríguez-Padilla C, Martínez-Rodríguez JL, Juárez-García DM, Sánchez-Armáss O, Sánchez T, et al. Psychological effects of group hypnotherapy on breast cancer patients during chemotherapy. Am J Clin Hipnosis 2017; 60: 68-84. doi: 10.1080 / 00029157.2016.1210497.

45. Jaime Bernal L, Téllez López A, Juárez García DM, García Cadena CH, García García E. El efecto de la hipnoterapia en la calidad de sueño de mujeres con cáncer de mama. Psicooncología 2015; 12: 39-49. doi: 10.5209/rev_PSIC.2015.v12.n1.48902

46. Lee V, Cohen SR, Edgar L, Laizner AM, Gagnon AJ. Meaning-making intervention during breast or colorectal cancer treatment improves self-esteem, optimism, and selfefficacy. Soc Sci Med 2006; 62: 3133-45. doi: 10.1016/j.socscimed.2005.11.041 